\title{
Big, old and complicated
}

\author{
Earth scientists learn to approach scientific questions from a unique perspective - one that \\ Charles Darwin shared.
}

\begin{abstract}
All geoscientists have an interest in some aspect of the Earth. But little else unites them at first glance: there is no canonical academic education that Earth scientists share - in sharp contrast to other subject areas. Almost everybody attending a physics conference will have a degree in physics, and thereby a common scientific grounding. At the largest Earth science assemblies, participants who studied a straightforward geoscience subject such as geology or geophysics are probably in the minority.

Instead, our conferences are populated with researchers from all disciplines of the natural sciences (plus some economists and sociologists). Ecologists who now work on the carbon cycle, meteorologists who started out as physicists, palaeontologists
\end{abstract}

with an interest in palaeoclimate, mathematicians turned climate modellers and chemists who decided to specialize in atmospheric chemistry or geochemistry all consider themselves Earth scientists. Charles Darwin - who is the centre of attention in this double-anniversary year marking his 200th birthday and the 150th anniversary of the publication of his On the Origin of Species - would have fitted into this diverse crowd very well.

Indeed, the commentary on page 666 and the two reviews in our Books and Arts section on pages 668-669 argue that Darwin thought like a geologist. According to a study on how geoscientists think and learn (Eos 90, 265-266; 2009), this means sharing "a distinctive set of approaches and perspectives that are particularly well-suited to studying something as big, old and complicated as the Earth system". More specifically, these approaches include "taking a long view of time, using temporal and spatial reasoning to formulate hypotheses and answer questions, interpreting observations in terms of intertwined processes rather than a single independent variable, and building cascades of inscriptions that begin with the raw materials of nature and tap into powerful visualization techniques."

If these attributes truly define an Earth scientist, then Charles Darwin was an exemplary representative of our multidisciplinary branch of science - even though his greatest work primarily revolutionized biology.

\section{Fake plastic trees}

\section{Greenhouse-gas emissions keep rising, despite all efforts at regulation and international agreement. Geoengineering could provide a back-up plan.}

Unless global greenhouse-gas emissions are controlled very tightly very soon, the planet is bound to become uncomfortably warm by the end of the century. The consequences could be chaotic and severe. Melting ice caps, rising sea levels and more frequent and intense floods, droughts and heat waves are all projected for the medium-term future. And the poorest nations are probably going to be hit worst. Drastic action - in the form of emissions reductions - is needed. And yet the world's nations have been desperately slow to act.

The goal of the United Nations climate change conference, due to take place in Copenhagen in December this year, is to devise an international strategy to follow the Kyoto protocol in confining climate change. But the willingness of the world's nations to commit to emissions cutbacks is unclear, given the disparate interests of the many participating countries (Nature doi:10.1038/461342a; 2009). Thus it seems essential to have a back-up plan. It is in this vein that the Royal Society launched a report on 1 September on the scientific merit and feasibility of the main geoengineering strategies.
The topic of geoengineering - the deliberate manipulation of the Earth's climate system to moderate global warming - is fraught with hysteria and uncertainty, primarily because of the sparseness of peer-reviewed literature on the subject, the colossal unknowns when it comes to consequences and the outlandishness of some of the ideas. Launching giant mirrors into space and filling the landscape with fake forests evokes images of a world gone awry. But given that our choices for avoiding the dangerous effects of climate change are running out, we can no longer afford to ignore engineering options.

The Royal Society report breaks down the proposed strategies into two camps - those that remove carbon dioxide from the atmosphere, and those that reflect sunlight back to space. The different schemes are ranked according to effectiveness, affordability, timeliness and safety (Nature Geosci. 1, 722-724; 2008). No overall winner emerges and, as the report stresses, there is no 'silver bullet'. But the evidence suggests that techniques that remove carbon dioxide from the atmosphere are preferable, as they involve fewer uncertainties and risks.
Of course there are many barriers, both scientific and legal, to the success of geoengineering techniques. At the report launch, Catherine Redgwell, Professor of International Law at University College London, stressed the urgent need for international governmental regulations to be put in place, pointing out that at present there is little to stop nations, corporations or even wealthy individuals pursuing some of these techniques. As of yet, there is no legal framework that fits all, but Redgwell suggested looking to schemes such as the London Convention, which regulates marine pollution (Nature Geosci. 2, 153; 2009).

It is not comforting that proposals to shade the planet and fertilize the oceans have to be taken seriously, but to ignore them because we do not like the idea of them would be short-sighted. Perhaps participants at the Copenhagen negotiations will map out a path for future greenhousegas emissions that is both effective and feasible. But given the uncertainties surrounding these negotiations, it is essential that we have a back-up plan. If geoengineering is that plan, it had better be well researched, well ahead of time. 